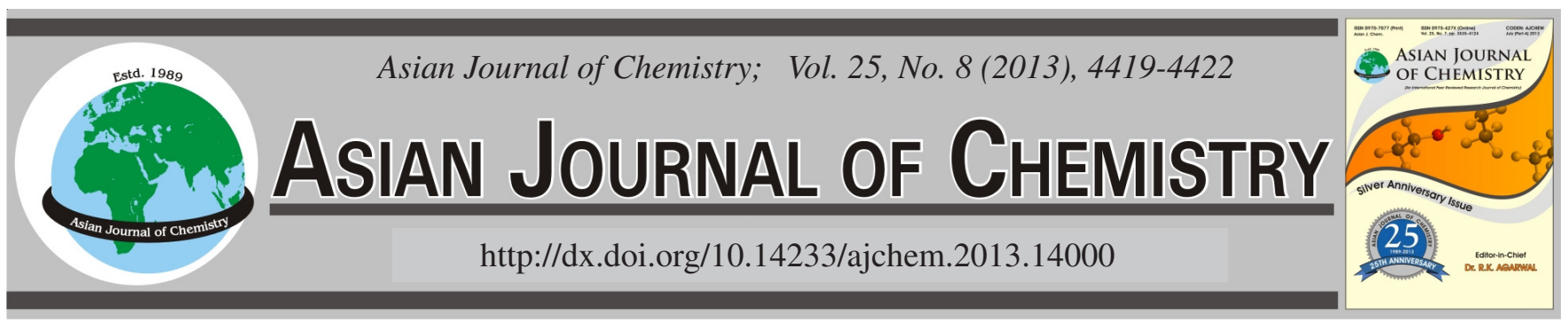

\title{
Synthesis and Characterization of Copolymer of Styrene and Acrylonitrile by Emulsion and Microemulsion Polymerization Using Different Emulsifiers
}

\author{
Farah Kanwal $^{1, *}$, Aisha Batool ${ }^{2},{\text { Madiha } \text { Yaseen }^{1} \text { and Muhammad Imran }}^{1}$
}

${ }^{1}$ Institute of Chemistry, University of the Punjab, Lahore, Pakistan

${ }^{2}$ Centre of Excellence in Solid State Physics, University of the Punjab, Lahore, Pakistan

*Corresponding author: Fax: +92 42 99231269; E-mail: farahkchem@yahoo.com

(Received: 30 April 2012;

Accepted: 13 February 2013)

AJC-12979

\begin{abstract}
Stable poly(styrene/acrylonitrile) (SAN) latexes were produced by polymerization of styrene and acrylonitrile in emulsion and microemulsion using sodium dodecyl sulfate and potassium oleate anionic emulsifiers. Their chemical structure, morphology and thermal behaviour were inspected by fourier transform infrared spectroscopy, scanning electron microscopy, thermo-gravimetric analysis and differential scanning calorimeter analysis respectively. Fourier transform infrared spectroscopy analysis verified the successful formation of copolymers of poly(styrene/acrylonitrile). The addition of different emulsifiers and acrylonitrile to the polystyrene improves its structure and thermal stability. Copolymer produced by microemulsion polymerization of styrene and acrylonitrile was found to be more thermally stable with higher molecular weight and molar mass and long range ordered microporous morphology with the reduction of particle size from 2-12 $\mathrm{nm}$ to $1-10 \mu \mathrm{m}$ as compared to other copolymers synthesized by emulsion polymerization.
\end{abstract}

Key Words: Microemulsion, Poly(styrene/acrylonitrile), Latexes.

\section{INTRODUCTION}

Microemulsion is a heterogeneous system of oil, water and amphiphilic compounds which is optically isotropic, transparent and thermodynamically stable liquid firstly introduced by Hoar and Schulman ${ }^{1,2}$. Emulsions and microemulsions are proficient to yield well defined latex particles with controllable high molecular weights. However the main difference between emulsions and microemulsions lies in the size and shape of the particles disseminated in the continuous phase. Particle nucleation in microemulsion polymerization of size smaller than 1-20 $\mu \mathrm{m}$ than those of conventional emulsion of size $2-100 \mathrm{~nm}$ is considered to be a continuous process and the locus of nucleation is shown to be the microemulsion droplet ${ }^{3,4}$ but exact mechanism of microemulsion copolymerization remained focused in the last decade by several authors ${ }^{5,6}$. Because of its smaller sized particles, thermodynamically optimized structure and unique solubilization properties, microemulsions have attracted increasing attention as potential drug delivery systems, either as vehicles for topical applications or as bioavailability enhancers for poorly water soluble active pharmaceutical ingredients and in dermal or transdermal drug delivery $^{7,8}$.

Poly(styrene/acrylonitrile) (SAN) is copolymer of styrene and acrylonitrile with superior mechanical properties, chemical and heat resistance as compared to polystyrene of general purposes. Copolymers of styrene and acrlyonitrile (SAN) are well known to have broad spectrum of applications such as automotive parts, battery cases, kitchenware, appliances, furniture and medical supplies due to their chemical, mechanical and optical nature. Furthermore they possess easy processibility and thermal stability. The copolymerization of these two monomers in bulk, solution or emulsion system has been widely reported ${ }^{9,10}$. Accessible approach through literature reveals that little work has been done to synthesize copolymer of styrene and acrylonitrile using emulsifier in microemulsion process $^{11,12}$. It has been observed that this type of polymerization in the presence of co-emulsifiers was complex, because they impart partition among different domains of microemulsions, monomers and aqueous phase and can change the location of monomers. It can modify the partitioning of monomer and may act as chain transfer agents ${ }^{13,14}$.

Keeping in attention in this complex copolymerization, we have synthesized the copolymer of styrene and acrylonitrile by emulsion and microemulsion polymerization by using two different emulsifiers. The variations in their properties have been investigated by using different analytical techniques.

\section{EXPERIMENTAL}

Styrene by Sigma-Aldrich chemie, acrylonitrile by Acros organics was distilled prior to use and kept under nitrogen. 
Sodium dodecylsulfate (SDS) and potassium oleate (PO) was purchased from Merck-Schuchardt. Potassium persulphate $\left(\mathrm{K}_{2} \mathrm{~S}_{2} \mathrm{O}_{3}\right)$ and sodium thiosulphate by $\mathrm{M}$ and $\mathrm{B}$ chemicals were used in this research work.

Synthesis of polystyrene and polyacrylonitrile: Monomers of styrene and acrylonitrile were washed firstly with $5 \%$ solution of $\mathrm{NaOH}$ to remove inhibitors and then distilled under reduce pressure and nitrogen flow. $0.5 \mathrm{M}$ solutions of sodium thiosulphate and ammonium persulphate were prepared in distilled water and then $40 \mathrm{~mL}$ of both solutions were added in $0.4 \mathrm{~g}$ of sodium dodecylsulfate. After mixing $20 \mathrm{~mL}$ of styrene or acrylonitrile was added in the above solution under constant magnetic stirring at $70^{\circ} \mathrm{C}$ for $4 \mathrm{~h}$. The reaction mixture was coagulated in $5 \% \mathrm{NaCl}$ solution and filtered under vacuum. The latex was washed by distilled water to remove surfactant and was dried by keeping in vacuum oven at $50{ }^{\circ} \mathrm{C}$ for $24 \mathrm{~h}$ to get polystyrene or polyacrylonitrile respectively ${ }^{15}$.

Synthesis of copolymers of styrene and acrylonitrile: Copolymers of styrene and acrylonitrile were prepared by adding $10 \mathrm{~mL}$ of both pure styrene and acrylonitrile monomers in different emulsifiers such as sodium dodecylsulfate, polystyrene and emulsifier free solutions to get SAN-I, SAN-2 and SAN-3 respectively as given in Table-1 and reflex at $70{ }^{\circ} \mathrm{C}$ for $4 \mathrm{~h}$. After reflex the reaction mixture was cooled and added to the $400 \mathrm{~mL}$ of $5 \% \mathrm{NaCl}$ solution until white polymer has formed. Copolymer was pressed and their salt solutions were decanted and collected samples were dried at $50^{\circ} \mathrm{C}$ for $24 \mathrm{~h}$.

\begin{tabular}{ccccc}
\multicolumn{5}{c}{ TABLE-1 } \\
\multicolumn{5}{c}{ CHEMICAL COMPOSITION OF ALL SAMPLES } \\
\hline $\begin{array}{c}\text { Sample } \\
\text { name }\end{array}$ & Emulsifier & $\begin{array}{c}\text { Styrene: } \\
\text { acrylonitrile }\end{array}$ & $\begin{array}{c}\text { Reaction } \\
\text { temp. }\left({ }^{\circ} \mathrm{C}\right)\end{array}$ & Copolymerization \\
\hline PS & SDS & $100: 0$ & 70 & Emulsion \\
PAN & SDS & $0: 100$ & 70 & Emulsion \\
SAN-1 & SDS & $50: 50$ & 70 & Emulsion \\
SAN-2 & PO & $50: 50$ & 70 & Emulsion \\
SAN-3 & $\times$ & $50: 50$ & 70 & Emulsion \\
SAN-4 & SDS & $50: 50$ & 70 & Microemulsion \\
\hline
\end{tabular}

Microemulsion polymerization: In microemulsion polymerization, the amount of emulsifier (sodium dodecyl sulfate) was increased from 0.4 to $1.5 \mathrm{~g}$. $10 \mathrm{~mL}$ of both styrene and acrylonitrile were added in the above reaction mixture. The microemulsion polymerization reaction was proceed at $70{ }^{\circ} \mathrm{C}$ for $4 \mathrm{~h}$ and latex was purified as described above and dried at $50{ }^{\circ} \mathrm{C}$ under vacuum ${ }^{16}$.

Characterization: IR-Prestige-21-fourier transfer infrared spectroscopic instrument operated at room temperature in the normal IR region $4000-650 \mathrm{~cm}^{-1}$ and $4 \mathrm{~cm}^{-1}$ band resolution was used for structural analysis. Polymers were dispersed in potassium bromide and compressed into pellets. S-3400 Hitachi scanning electron microscope was used for morphological analysis. Scanning electron microscope was operated in back scattered electron image mode. All images were obtained at an accelerating voltage of $25 \mathrm{KV}$. Thermal analysis of polystyrene, polyacrylonitrile and all SAN samples was studied by SDT Q-600 TGA/DSC instrument in the temperature range of $25^{\circ} \mathrm{C}$ to $1500{ }^{\circ} \mathrm{C}$ and at heating rate of $10^{\circ} \mathrm{C} / \mathrm{min}$ under inert atmosphere. Differential scanning calorimetry measurements were performed in Q-200 DSC, TA instrument from 25 to $400^{\circ} \mathrm{C}$ with refrigerated cooling system $(\mathrm{RCS})$ at $10^{\circ} \mathrm{C} / \mathrm{min}$ under inert atmosphere. Ostwald viscometer was used to determine molecular masses of all samples. Dimethylformamide (DMF) was used as a solvent and molar masses of all samples have been determined by viscosity method at $25^{\circ} \mathrm{C}$ by using Mark Hounwink equation:

$$
[\eta]=\mathrm{k} \mathrm{M}^{\mathrm{a}}
$$

where $\mathrm{k}=1.2 \times 10^{-4}$ and $\mathrm{a}=0.77$

\section{RESULTS AND DISCUSSION}

FT-IR analysis: Fig. 1 shows the FT-IR spectrum of polystyrene that exhibits the characteristic frequency of aromatic $\mathrm{C}-\mathrm{H}$ stretching at $3107 \mathrm{~cm}^{-1}$, aliphatic C-H stretching at 2771 $\mathrm{cm}^{-1}$, aromatic ring of styrene at $1523 \mathrm{~cm}^{-1}$ and the peak of $\mathrm{C}-\mathrm{H}$ bending of mono-substituted ring at $694 \mathrm{~cm}^{-1}{ }^{17}$. Polyacrylonitrile shows the characteristic vibrational bands at 2883 $\mathrm{cm}^{-1}$ for aliphatic C-H stretching and $2304 \mathrm{~cm}^{-1}$ which are assigned to $-\mathrm{C}=\mathrm{N}$ stretching vibration, while the peak appeared at $1413 \mathrm{~cm}^{-1}$ is due to $\mathrm{CH}_{2}$ scissoring mode as shown in Fig. 1 (b) ${ }^{18}$. FTIR spectra of SAN-1, SAN-2, SAN-3 and SAN-4 also exhibit the characteristic frequency of $-\mathrm{C}=\mathrm{N}$ stretching at 2334 , 2338, 2339 and $2339 \mathrm{~cm}^{-1}$, aliphatic C-H stretching at 2839, 2960, 2920 and $2960 \mathrm{~cm}^{-1}$ respectively. Characteristic peak of aromatic ring of styrene appeared almost at $1530 \mathrm{~cm}^{-1}$ in all SAN copolymers, $\mathrm{C}-\mathrm{H}$ bending and $\mathrm{CH}_{2}$ scissoring modes appeared in the range of $710-650 \mathrm{~cm}^{-1}$ and $1400-1350 \mathrm{~cm}^{-1}$, respectively. Band showing $\mathrm{C}-\mathrm{N}$ stretching and aromatic ring is the characteristic bands of SAN copolymers ${ }^{19}$.

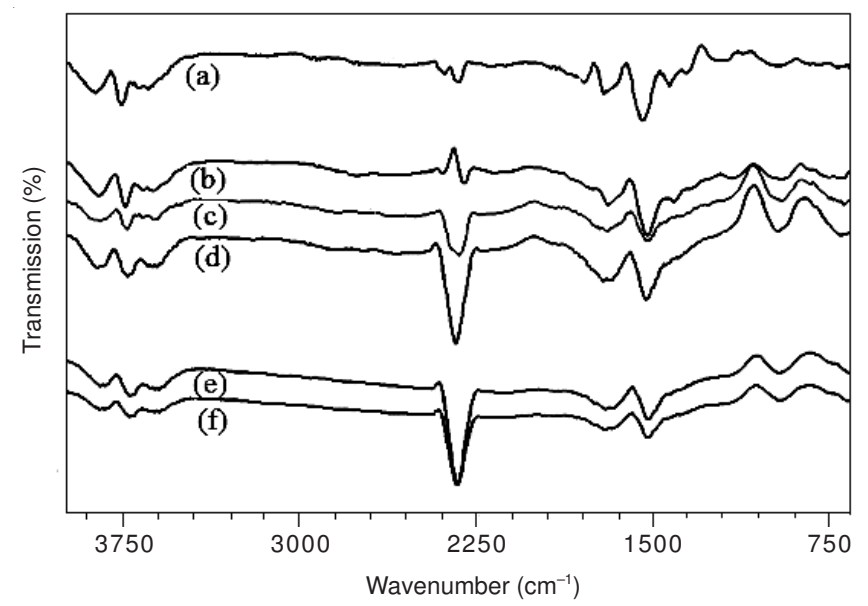

Fig. 1. FTIR spectra of (a) PAN (b) PS (c) SAN-1 (d) SAN-2 (e) SAN-3 (f) SAN-4

Percentage yield and molar mass determination: The percentage yield and molar mass of all SAN samples indicates that molecular weight of all copolymers is dependent on composition and nature of used emulsifiers (Table-2). It is also cleared from results that copolymer of SAN synthesized under microemulsion polymerization offered highest yield and molar mass among all prepared samples.

Scanning electron microscopy analysis: Fig. 2a shows the SEM micrograph of SAN-1 in which rectangular shaped particles of size 2-12 nm are seen to be bounded firmly with each other in a highly blended material. In SAN-2 particles of irregular shape and the size in the range of $10-35 \mu \mathrm{m}$ are 
scattered to some extent in the form of long range small branches on the surface. Grains of SAN-3 have much irregular shape and particles are not oriented in a well-defined way. It may be due to the fact that no emulsifier has been used during fabrication that could bind the particles together. Fig. 2d shows the copolymer of SAN synthesized in microemulsion polymerization having microporous pronged morphology with clear and denser branched shape assembly with small branches attached to long ones. They are elongated up to more than 500 $\mu \mathrm{m}$ length to form a continuous phase with particles of size $1-10 \mu \mathrm{m}$ bounded with each other in an interesting way.

\begin{tabular}{ccc}
\multicolumn{3}{c}{ TABLE-2 } \\
\multicolumn{3}{c}{$\begin{array}{c}\text { MOLAR MASS AND PERCENTAGE YIELD } \\
\text { OF COPOLYMERS OF SAN }\end{array}$} \\
\hline Sample & Yield $(\%)$ & Mol. mass $\left(10^{5} \mathrm{~g} / \mathrm{mol}\right)$ \\
\hline SAN-1 & 82.06 & 0.11 \\
SAN-2 & 47 & 1.02 \\
SAN-3 & 35.3 & 0.91 \\
SAN-4 & 92 & 2.09 \\
\hline
\end{tabular}
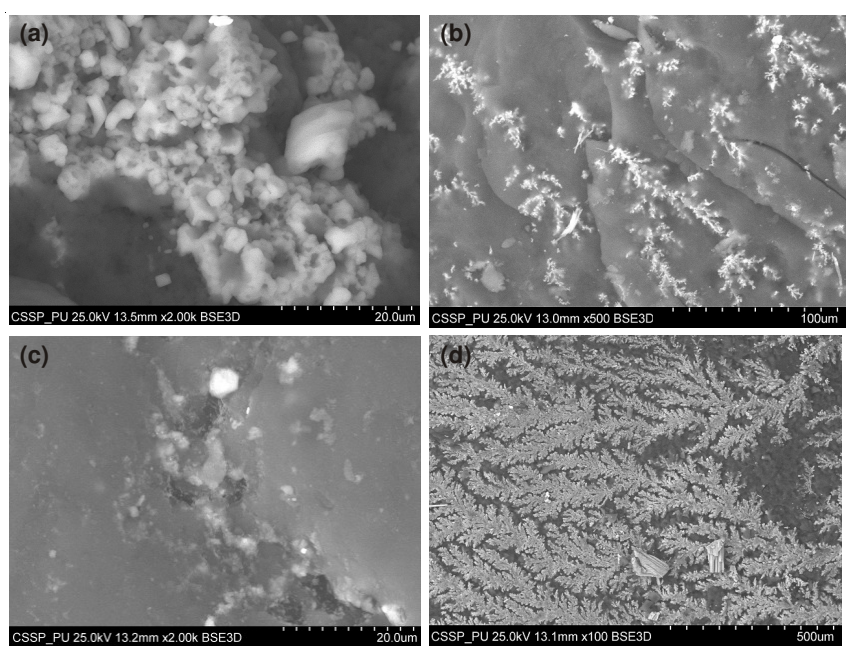

Fig. 2 SEM images of (a) SAN-1 (b) SAN-2 (c) SAN-3 (d) SAN-4

Thermal gravimetric analysis: Thermal degradation of polystyrene, polyacrylonitrile and their copolymers have been studied in an inert atmosphere and the percentage weight loss as the function of temperature for all samples are given in Table-3 (Fig. 3). The TGA thermograms of polystyrene and polyacrylonitrile (PAN) exhibited two-step weight loss in the range of $25-475^{\circ} \mathrm{C}$. Weight loss in first step is assigned to the loss of moisture and in second step due to the degradation of copolymer ${ }^{20,21}$. Polyacrylonitrile was found to be more thermally stable than polystyrene with $57.24 \%$ weight loss from $290-470{ }^{\circ} \mathrm{C}$, which degrades $86 \%$ in that given temperature range. Above $500{ }^{\circ} \mathrm{C}$ the weight loss is very slow this is due to the char-oxidation of polyacrylonitrile residues ${ }^{22}$. While copolymer of styrene and acrylonitrine revealed one step thermal degradation initiated from $340{ }^{\circ} \mathrm{C}$ and decomposed completely at $435{ }^{\circ} \mathrm{C}$ with $94.86 \%$ weight loss. SAN-2 and SAN-3 also followed the similar trend of two zones of weight loss like polystyrene and polyacrylonitrile. The first weight loss of 7.37 and $16.95 \%$ and second weight loss of 80 and 82 $\%$ for SAN-2 and SAN-3 respectively as given in Table-3. Microemulsive copolymer showed maximum thermal stability among all copolymers with the weight loss of only $75.83 \%$ in one step like SAN-1 in the temperature range of 300 to $460{ }^{\circ} \mathrm{C}$ that can be attributed to the long range continuous phase of micro latex particles nucleation ${ }^{23,24}$.

\begin{tabular}{ccccc}
\multicolumn{5}{c}{ TABLE-3 } \\
\multicolumn{5}{c}{$\begin{array}{c}\text { DSC AND TGA DATA OF PS, PAN, SAN-1, } \\
\text { SAN-2, SAN-3 AND SAN-4 }\end{array}$} \\
\hline Sample & $\begin{array}{c}\text { Onset Temp. } \\
\left({ }^{\circ} \mathrm{C}\right)\end{array}$ & $\begin{array}{c}\text { Ending } \\
\text { Temp. }\left({ }^{\circ} \mathrm{C}\right)\end{array}$ & $\begin{array}{c}\text { Weight } \\
\text { loss }(\%)\end{array}$ & $\mathrm{Tg}\left({ }^{\circ} \mathrm{C}\right)$ \\
\hline PS & 350 & 490 & 34.17 & 100 \\
PAN & 240 & 450 & 87.38 & 98 \\
SAN-1 & 240 & 460 & 86.31 & 112 \\
SAN-2 & 270 & 500 & 85.91 & 109 \\
SAN-3 & 290 & 460 & 83.79 & 88 \\
SAN-4 & 300 & 500 & 75.83 & 114 \\
\hline
\end{tabular}

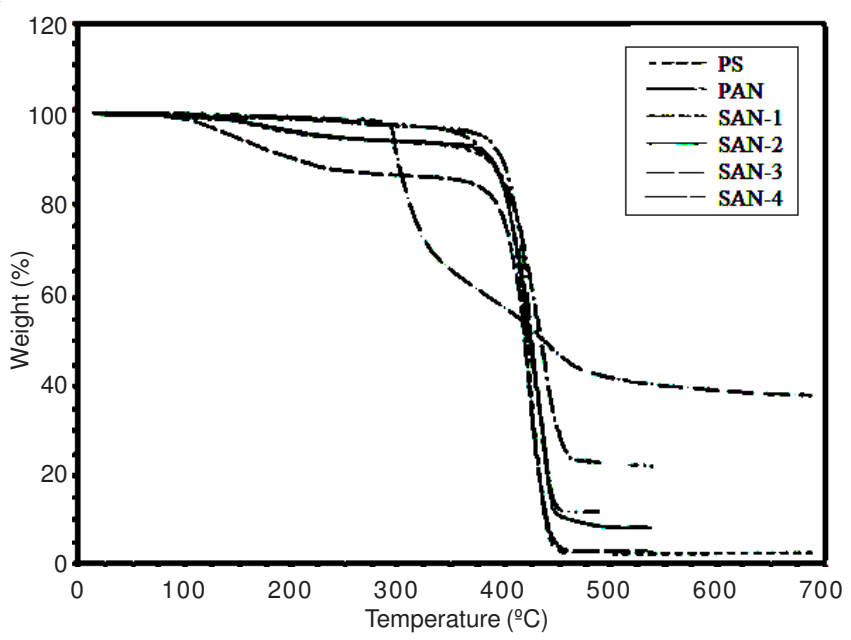

Fig. 3. TGA thermograms of PS, PAN, SAN-1, SAN-2, SAN-3, SAN-4

Differential scanning calorimeter analysis: The DSC themograms of polystyrene, polyacrylonitrile and all SAN samples are depicted in Fig. 4. Polyacrylonitrile showed an endothermic curve in heat flux at $95^{\circ} \mathrm{C}$ due to transformation from amorphous glassy phase to a flexible substance and an exothermic curve at initial stabilization temperature 300.66 ${ }^{\circ} \mathrm{C}$, due to its glassy nature contributed towards the cyclization of nitrile group contributed to the evolution of large amount of heat released from the polyacrylonitrile homopolymer ${ }^{21,22,24}$. It can be seen that glass transition temperatures of all copolymers except SAN-2 are shifted towards higher temperatures as compared to the pure polystyrene and polyacrylonitrile as shown in Table-3. This decrease in glass transition temperature of SAN-2 may be attributed to the higher symmetry and low chain rigidity offered by potassium oleate emulsifier to lower its thermal stability ${ }^{25}$.

Continuing to heat copolymers former to its glass transition temperature eventually leads to another endothermic transition peak due to melting of polystyrene in the range of $350-490{ }^{\circ} \mathrm{C}$. All samples were found to be more thermally stable than polystyrene but the microemulsive SAN presented highest glass transition and melting temperatures of $114^{\circ} \mathrm{C}$ and $440.54{ }^{\circ} \mathrm{C}$ due to bulky chains, higher molecular weight, molar mass and crosslinking as indicated by viscometer and SEM studies. 


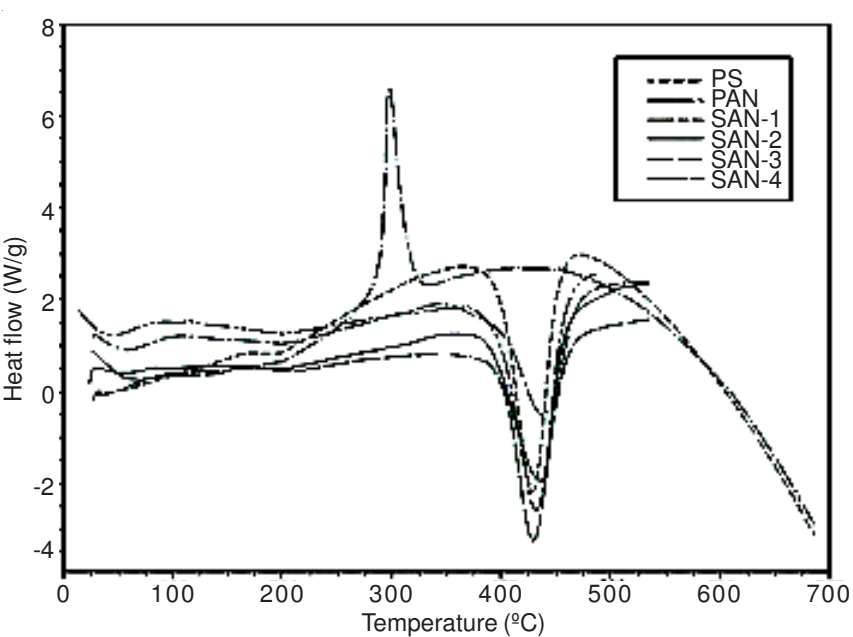

Fig. 4. DSC thermograms of PS, PAN, SAN-1, SAN-2, SAN-3, SAN-4

\section{Conclusion}

Copolymers of styrene and acrlyonitrile are successfully prepared by emulsion and microemulsion polymerization by using two different emulsifiers. Copolymer produced under microemulsion exhibit dramatically enhanced structural and thermal properties among all other prepared samples due to its higher molecular weight, molar mass, micro-porous particles and bulky nature as inferred from viscometer and SEM studies. Glass transition temperature was also explored to depend on the molecular weight of copolymer.

\section{ACKNOWLEDGEMENTS}

This work was supported by Institute of Chemistry and Centre of Excellence in Solid State Physics, University of the Punjab, Lahore, Pakistan.

\section{REFERENCES}

1. J.O. Stoffer and T. Bone, J. Polym., 39, 187 (2007).

2. I. Capek and V. Juranicova, J. Polym. Sci., 34, 575 (1996).

3. N.K. Pokhriyal and S. Devi, Eur. Polym. J., 36, 333 (2000).

4. D. Donescu, M. Vasilescu, L. Fusulan, C. Petcu and L. Fusulan, Langmuir, 15, 27 (1999).

5. D. Donescu, L. Fusulan, C. Petcu, A.G. Boborodea and D.S. Vasilescu, Eur. Polym. J., 37, 1499 (2001).

6. D.J. Hill, J.H. Donnell and P.W. Sullivan, Macromolecules, 15, 960 (1982).

7. J.I. Asakura, J. Macromol. Sci. Chem. Soc., 15, 1473 (1981).

8. J. Guillot, Acta Polym., 32, 593 (1981).

9. K.C. Lee, L.M. Gan and C.H. Chew, Polym. J., 36, 3719 (1995).

10. P.G. Sanghvi, A.C. Patel, K.S. Gopalkrishnan and S. Devi, Eur. Polym. J., 36, 2275 (2000).

11. J.R. Herrera, R.D. Peralta, R.G. Lopez, L.C. Cesteros, E. Mendiza-bal and J.E. Puig, Polymer, 44, 1795 (2003).

12. C.S. Chern and C.W. Liu, Colloid Polym. Sci., 278, 329 (2000).

13. C.S. Chern and L.J. Wu, J. Polym. Sci., 39, 898 (2001).

14. C.S. Chern and L.J. Wu, J. Polym. Sci., 39, 3199 (2001).

15. S.-L. Tsaur and R.M. Fitch, J. Colloid Interf. Sci., 115, 462, (1987).

16. Y. Shi, Y. Wu, J. Hao and G. Li, J. Polym. Sci., 43, 216 (2005).

17. Z. Wang and Y.P. Ting, Ind. Eng. Chem. Res., 47, 1867 (2008).

18. H. Bu, J. Rong and Z. Yang, Macromol. Rapid Commun., 23, 464 (2002).

19. M.H. Noh and D.C. Lee, J. Appl. Polym. Sci., 74, 2819 (1999).

20. Y. Shimura, J. Polym. Sci., 4, 435 (1966).

21. C.R. Martines and G. Ruggeri, J. Brazil. Chem. Soc., 14, 5 (2003).

22. S. Yang, J.R. Castilleja, E.V. Barrera and K. Lozano, Polym. Degrad. Stab., 83, 383 (2004).

23. J.-H. Shi, B.-X. Yang and S.H. Goh, Eur. Polym. J., 45, 1008 (2009).

24. Y.S. Choi, M. Xu and I. Chung, J. Polym., 44, 6994 (2003).

25. N. Yusof and A.F. Ismail, Int. J. Chem. Environ. Eng., 1, 79 (2010). 